\title{
Advances in gastroenterology and hepatology
}

\author{
M C Bateson
}

\begin{abstract}
This is a review of some of the most important growing points in the specialties of gastroenterology and hepatology. It does not aim to be completely comprehensive but to pick out major areas of importance to examination candidates and doctors without special experience in the field.

Topics covered include: upper gastrointestinal haemorrhage; Barrett's oesophagus; carcinoma of the oesophagus; achalasia; Helicobacter pylori; duodenal ulcer prevention; coeliac disease; dermatitis herpetiformis; Crohn's disease; small bowel overgrowth; ulcerative colitis; carcinoma of the large bowel; obesity; endoscope sterilisation; gall stones; liver transplantation; autoimmune liver disease; viral hepatitis; metabolic liver diseases; and pancreatic insufficiency. (Postgrad Med F 2000;76:328-332)
\end{abstract}

Keywords: gastroenterology; hepatology

Upper gastrointestinal haemorrhage

Bleeding peptic ulcers can be controlled by various endoscopic means, including laser therapy and heater probes, but injection treatment is cheap, simple, and satisfactory. Adrenaline (epinephrine) at 1 in 10000 in several 1-2 $\mathrm{ml}$ boluses will stop bleeding. Addition of sclerosants does not confer additional efficacy. Acrylic glues tend to gum up the equipment, but thrombin has shown promise as an adjuvant to adrenaline. Unfortunately there is a big question mark over the introduction of new uses for human blood products, and we should probably await trials of synthetic thrombin. These procedures have much reduced reliance on open surgical intervention.

For oesophageal varices banding (using the Saaed six shooter) is now proved to be a better technique than injection sclerotherapy.

Transvenous intrahepatic portasystemic stent shunting (TIPS) for decompression of the portal venous system is also used to control of oesophageal variceal bleeding.

There are many other standard procedures such as formal portasystemic anastomosis, liver transplant, and drug treatment with propranolol, isosorbide mononitrate, and intravenous terlipressin. The usefulness of octreotide in variceal bleeding is still in doubt with a host of contradictory studies.

TIPS does not prolong life, but patients succumb to hepatic encephalopathy rather than gastrointestinal bleeding. It is ineffective in control of ascites as problems outweigh benefits.

\section{Barrett's oesophagus}

It is more than 40 years since Barrett first described columnar lined oesophagus. He mis- takenly thought this was a congenital entity, but it is now generally accepted that it is the result of chronic gastro-oesophageal reflux disease. There has been renewed interest in this condition recently and a British National Barrett's Oesophagus Registry has been set up in an attempt to define the natural history of the condition.

One of the problems is that of definition, in what is a clinicopathological concept. The endoscopist is usually readily able to recognise when the transition from pink squamous epithelium to red columnar epithelium occurs more than $3 \mathrm{~cm}$ proximal to the junction of the tubular oesophagus and the gastric cardia. However, in addition to this macroscopic finding it is generally believed that histological proof should be obtained with a minimum of two biopsies taken from the area.

This immediately raises a problem of interpretation. The finding of gastric metaplasia satisfies the definition of columnar lined epithelium, but many believe that actual intestinal metaplasia should be demonstrated before accepting the diagnosis. Worse than this, changes can be patchy so that some areas of Barrett's mucosa are squamous, some show gastric metaplasia and some intestinal metaplasia. The length of columnar lined oesophagus required to establish the diagnosis has also been subject to controversy, and the expression "short segment Barrett's oesophagus" has been introduced for patients who have histological intestinal metaplasia in the distal oesophagus less than $3 \mathrm{~cm}$ above the gastro-oesophageal junction. Furthermore biopsies at the gastrooesophageal junction and in the gastric cardia also sometimes show intestinal metaplasia and this has been termed "ultra short segment Barrett's oesophagus".

No drug or surgical therapy alters the length or histology of Barrett's mucosa, but permanent full dose proton pump inhibitor therapy is advised to control symptoms.

Barrett's oesophagus may be premalignant, and this is particularly true in long segment disease where more than $7 \mathrm{~cm}$ is affected, and when dysplasia is demonstrated on biopsy. These two conditions warrant periodic endoscopic surveillance in their own right, though there is absolutely no evidence that routine endoscopic surveillance benefits patients overall.

Where dysplasia or early carcinoma occur a recently introduced mode of treatment may be successful. After priming with a systemic porphyrin derivative argon plasma laser therapy may be used to produce focal superficial tissue destruction. This may cure early lesions and also control dysphagia in advanced lesions where other modes of treatment cannot be used or are not completely successful. 
Carcinoma of the oesophagus

This is increasing in frequency, particularly adenocarcinoma. There have been some developments in management. Aggressive and meticulous surgical resection now yields much better long term survival results and reduced operative mortality. There is much interest in combination modes of treatment, particularly using neoadjuvant therapy, where combination chemotherapy and radiotherapy are given before surgery. Staging of oesophageal carcinomas has become more refined with the use of endoscopic ultrasonography, which is becoming a standard procedure in specialised centres. More exact three dimensional mapping of tumours allows much better targeting of radiography treatment, so called conformal radiotherapy.

\section{Achalasia}

As well as the traditional use of balloon dilation and surgical cardiomyotomy, achalasia can now be managed by botulinum toxin injections. At endoscopy 25 units of botulinum toxin are placed in each of the four quadrants at the level of the lower oesophageal sphincter. This can yield relief of symptoms for some months and can be repeated as often as required. It does not cure the condition but may give good symptomatic relief where other modes of treatment cannot be used. Where surgical correction is required then laparoscopic myotomy is yielding promising results.

\section{Helicobacter pylori}

In addition to its strong association with peptic ulcer disease, Helicobacter pylori is now recognised as a carcinogen, and is linked with the development of adenocarcinoma in the distal stomach. However, it is important to remember that overall prevalence of adenocarcinoma of the stomach has been falling for decades. There has been a particularly sharp fall in distal carcinoma of the stomach concurrent with a rise in carcinoma of the proximal stomach and gastro-oesophageal junction, which are definitely not positively associated with $H$ pylori, and may even be negatively associated.

Though $H$ pylori has been associated with many other conditions including ischaemic heart disease, migraine and gall stones, these postulated links are dubious.

Long term cure of peptic ulcer disease may be achieved by eradication of $H$ pylori. Successful modern treatments include a proton pump inhibitor (PPI) such as omeprazole 20 $\mathrm{mg}$ once or twice daily, lansoprazole $30 \mathrm{mg}$ once or twice daily or pantoprazole $40 \mathrm{mg}$ daily, together with two or three antibiotics. The most successful regimens are those which include clarithromycin 250 to $500 \mathrm{mg}$ twice daily, and either amoxycillin $1000 \mathrm{mg}$ twice daily or metronidazole $400 \mathrm{mg}$ twice a day.

Alternative regimens include the use of ranitidine bismuth citrate $400 \mathrm{mg}$ twice a day instead of a PPI, and quadruple therapy using a PPI and either bismuth plus two antibiotics or three antibiotics.

Antibiotic treatment need only be given for a week to achieve optimal results. It is still unclear whether PPI and bismuth treatment should be continued for a further three weeks to get best early healing results.

The newest PPI, rabeprazole, is not yet licensed for antihelicobacter therapy, and the required doses for this and for oesophagitis are unclear. However, rabeprazole $20 \mathrm{mg}$ daily is the cheapest PPI for primary duodenal ulcer healing.

\section{Duodenal ulcer prevention}

With control of H pylori an increasingly important emerging problem has been iatrogenic ulcers. Aspirin, even in small doses, can cause severe gastroduodenal damage. Where this has occurred then an alternative antiplatelet agent is clopidogrel $75 \mathrm{mg}$ daily. All of the nonsteroidal anti-inflammatory drugs (NSAIDs) are prone to cause peptic ulceration. Azapropazone and piroxicam appear especially prone to do so. By contrast meloxicam and etodolac have been promoted as safer.

The benefits of NSAID treatment relate to inhibition of cyclo-oxygenase 2 (COX2), whereas the gastrointestinal side effects relate to activity against cyclo-oxygenase I. Drugs having specific action against COX 2 are being introduced (rofecoxib is the first) and may make the life of a gastroenterologist a little easier. This is being assessed by the National Institue for Clinical Evidence (NICE).

\section{Coeliac disease and dermatitis herpetiformis}

Intolerance of gluten was described during the second world war as the cause of these conditions, and very gratifying results have been achieved by exclusion diets. Traditionally these have excluded cereals such as wheat, rye, barley and oats, but allowed rice and maize (so that patients can eat Corn Flakes!). However, the chemistry of cereal protein in oats is quite different from rye, barley and wheat, and foods such as porridge which are derived from this source are almost certainly safe. There needs to be a little caution, however, because millers may process more than one sort of cereal and there may be contamination of oats with truly allergenic cereals.

Diagnosis of coeliac disease has been improved by the description of the frequent association with $\operatorname{IgA}$ endomysial antibody, though distal duodenal or jejunal biopsy will still be needed to prove diagnosis. Endomysial antibody is fairly specific for coeliac disease, though sensitivity in adults may be only around $70 \%$. Tissue transglutaminase is the autoallergen for endomysial antibody, and this may offer potential for a more specific test in future.

\section{Crohn's disease}

This really does seem to be increasing in frequency both in children and adults. Cigarette smoking clearly predisposes to the disease and stopping smoking reduces frequency and severity of relapses.

Conventional management with steroid therapy has been bedevilled by side effects and the introduction of budesonide is a useful manoeuvre to limit these. Budesonide is 
normally given in courses of $2-3$ months starting at a dose of $9 \mathrm{mg}$ daily. This will act in the gut but $90 \%$ is broken down to inactive metabolites in the liver on first pass through the portal circulation, so that systemic effects are much less than with prednisolone. However, they are not completely absent and undesired weight gain in particular may be a feature of budesonide use.

Though it is usual to discontinue azathioprine maintenance therapy in pregnancy, and where this is planned, if patients require this then there is no appreciable teratogenic effect. Alternative immunosuppressant treatment with cyclosporin by mouth has been disappointing. Mycophenolate has also been proposed, but has important toxic side effects.

Possible association with Mycobacterium paratuberculosis has led to new ideas in treatment.

Experimental use of antileprosy treatment (clofazimine, clarithromycin, rifabutin) has been reported to yield good results in some patients.

Research into the mechanisms of Crohn's disease has focused on the damaging effect of tumour necrosis factor-alpha (TNF- $\alpha$ ). This led to the development of a chimeric monoclonal antibody to TNF- $\alpha$ (infliximab or cA2). This is given as up to three infusions in a dose of $5 \mathrm{mg} / \mathrm{kg}$ and produces control of active disease and healing of fistulas in about two thirds of patients. Not least, thalidomide has recently been favourably assessed, but obviously requires caution in view of its notorious teratogenic effects.

\section{Small bowel overgrowth}

Bacterial colonisation of the proximal intestine is increasingly recognised as a cause of malnutrition in the elderly. The ${ }^{14} \mathrm{C}$-glycocholate breath test has been used as a non-invasive way of assessing this, but the ${ }^{14} \mathrm{C}$-xylose breath test has been promoted as a more effective technique. Where there are positive results treatment for two weeks with combination antibiotics such as metronidazole and amoxycillin can lead to nutritional improvement.

\section{Ulcerative colitis}

Oral budesonide may have a role in total or extensive ulcerative colitis but this is not yet completely established. Topical budesonide by enema or foam is available for left sided colitis and proctitis, and promoted as avoiding systemic steroid side effects. However, these are not generally a problem because of the way that topical steroid therapy is used in ulcerative colitis. Particularly where prednisolone sodium metasulphobenzoate retention enemas and foams are used absorption is not a clinical problem.

An activity index has recently been described using mathematical scoring for clinical features of ulcerative colitis. This may be useful in research projects but it is not yet apparent that it contributes more than simple weighing patients, questioning about diarrhoea and wellbeing, and measuring a full blood count.
Lively debate continues over the role of colonoscopy screening in ulcerative colitis. Although extensive and total ulcerative colitis are premalignant conditions, they account for only about $1 \%$ or fewer of large bowel carcinomas. Traditionally colonoscopy screening every one to two years has been felt useful for ulcerative colitis patients, though there are immense practical difficulties in operating this policy consistently. A very disappointing 11 year follow up study from Scandinavia indicated that overall mortality was actually increased in ulcerative colitis patients who underwent colonoscopy screening compared with controls. The excess mortality related to heart disease and accidents. It appeared that patients in the screening programme falsely believed their health was globally protected, and so indulged more in risky behaviour such as smoking and careless driving.

The old Truelove regimen for active ulcerative colitis has seen some very important recent modifications. High dose steroid therapy remains a mainstay of treatment, but it is now clear that patients do not need to fast, so that oral mesalazine and antibiotics can be used as appropriate. Mesalazine is now available as a foam or enema. It is not tolerated by all patients but certainly does benefit some. Two recent innovations in drug treatment have been introduced. Cyclosporin intravenously $4 \mathrm{mg} / \mathrm{kg}$ daily for a week is useful in refractory acute ulcerative colitis. Some authors have preferred to use smaller doses but these may be less effective. Treatment for longer than a week is not yet validated. A particularly interesting development is the use of full dose unfractionated heparin to maintain the prothrombin ratio between 1.5 and 2. This rather paradoxical therapy probably works through its antiinflammatory action. Its overall usefulness is currently the subject of a controlled trial in the north east of England. It is unclear whether low molecular weight heparin is equally effective.

Where surgery is required small bowel pouches have been popular to avoid permanent ileostomy, but do have drawbacks including a $30 \%$ prevalence of pouchitis. Ileostomy is still the best option for many patients.

\section{Carcinoma of the large bowel}

Results of treatment remain rather disappointing and effects are being made to achieve earlier diagnosis and better staging to focus therapy more precisely. Magnetic resonance imaging (MRI) is superior to conventional computed tomography, being able to do smaller liver metastases and better images in the pelvic colon. With the development of spiral computed tomographic scanning it has been possible to perform what has been called virtual colonoscopy with air insufflation into the large bowel. This pneumocolography looks very promising and may be able to replace barium enema and MRI in the future.

Screening the general population has become a politically fashionable topic. Occult blood testing schemes have some role, but are very labour intensive and throw up a lot of false positives. An alternative approach is offering a 
flexible sigmoidoscopy to the population between the ages of 50 and 55 . This is currently under formal evaluation in Britain, but is already included in some commercial "health" elective medical examinations of the ostensibly fit.

Cancer risk in families can be partly identified by screening for mismatch repair genes. Routine five yearly colonoscopy in families with hereditary non-polyposis colon cancer should detect cases early enough to prevent cancer.

The possibility of prophylaxis of large bowel carcinoma has generated many interesting ideas. The prevalence of the disease appears to be lower in patients on treatment with folate, aspirin, NSAIDs, and with ursodeoxycholic acid therapy of chronic liver disease. Of these folate supplementation and aspirin therapy offer the most promise for formal long term review. Antioxidant treatment with vitamins A, $\mathrm{C}$, and $\mathrm{E}$ and high fibre diets also have theoretical attractions. Cabbage, sprouts and broccoli contain oltipraz, and grapes, strawberries, raspberries and nuts contain ellagic acid, both of which may have anticancer potential.

Despite earlier fears, azathioprine maintenance therapy in ulcerative colitis does not increase bowel carcinoma prevalence, and may even be protective. It is clear that regular aminosalicylate therapy is strongly protective.

\section{Obesity}

Just when we had reached the point where all antiobesity drugs were being officially discouraged, orlistat has been introduced. This is not systemically active but inhibits intestinal activity of pancreatic lipase and produces a mild form of steatorrhoea. In addition to an energy controlled diet it has improved weight loss, but caution is required before it is used long term. Previous experience has shown that patients tend to believe that drug treatment will allow them to eat what they please, so they lose all the benefits.

Orlistat is under consideration by NICE.

\section{Endoscope sterilisation}

Sensitisation to glutaraldehyde is a problem affecting about one third of staff on gastroenterology units. This is the only generally recognised agent which is effective against bacterial spores and hepatitis B and C in the UK. Alternatives such as peracetic acid and $70 \%$ alcohol have been proposed. Seven per cent hydrogen peroxide is now officially recognised as an alternative to glutaraldehyde in the United States and may be the best option in the future to protect the health of staff.

\section{Gall stones}

The last 10 years has seen laparoscopic cholecystectomy emerge as the procedure of choice, and it now accounts for two thirds of gall bladder operations in Britain. However, it has also led to an increase in the frequency of surgery, which is now being offered to an older and less fit population. Consequently neither the overall morbidity or mortality of gall bladder surgery has changed. Hospital beds are used more intensively with shorter inpatient stays and consequently costs have not fallen.

\section{Liver transplantation}

In addition to the very satisfactory results obtained in children, in primary biliary cirrhosis and in paracetamol poisoning, improved outcomes are now being seen in primary sclerosing cholangitis (PSC). Two difficulties in PSC that previously compromised success were large bile duct strictures and the difficulty of identifying cholangiocarcinoma. Modern imaging techniques, especially the rarefied positron emission tomography for identifying cholangiocarcinoma, have helped in better selection of candidate patients.

After about three months the survival of transplant patients in general parallels that of the whole population.

Alcoholic liver disease in those who have abstained for six months is now a standard indication for transplantation and it is possible to demonstrate survival equivalent to other groups transplanted.

The main limitation on transplant numbers is now the inadequate supply of donors.

Various strategies have been proposed to remedy this problem. Use of living related donors and split liver grafts, where one donor liver is transplanted into two recipients, may offer a small increase in the number of patients treated.

What would be much more useful would be the adoption of an "opt-out" system of organ harvesting instead of the present inhibiting system. This would mean that every suitable donor could be utilised unless they had specifically expressed a wish to the contrary in life, or if their relatives had objections after death.

\section{Autoimmune liver disease}

Primary biliary cirrhosis has traditionally required liver biopsy for proof and histological staging. With the advent of testing for M2 fraction of antimitochondrial antibody we have available a specific test that may avoid the need for diagnostic histology. This is particularly important where clotting abnormalities make liver biopsy potentially hazardous. Ursodeoxycholic acid $750 \mathrm{mg}(13 \mathrm{mg} / \mathrm{kg})$ daily is now standard treatment for patients with primary biliary cirrhosis who are not in end stage disease or imminently awaiting transplant. It has been shown in several separate studies to improve survival, though the evidence is only grade $\mathrm{B}$ or $\mathrm{C}$.

Ursodeoxycholic acid has also been used in primary sclerosing cholangitis but with less encouraging results. The only other drug of which there is extensive experience in primary sclerosing cholangitis is methotrexate, and severe pulmonary complications have very much limited the use of this agent in liver disease. Management of primary sclerosing cholangitis is likely to need to be based on intermittent antibiotic therapy, balloon dilation and stenting of strictures, and liver transplantation in selected patients.

Despite earlier concepts that osteomalacia would be the dominant bone problem in chronic liver disease because of vitamin D malabsorption, this has not proved to be the case. Osteoporosis is a much more important 
difficulty. Etidronate plus calcium can help prevent bone demineralisation. It has recently become clear that hormone replacement therapy, especially where oestrogens are given percutaneously, is probably safe in women with primary biliary cirrhosis, and should be considered. Calcium plus vitamin D may also be useful.

Chronic autoimmune hepatitis (CAIH) is now divided into type I, associated with nuclear and smooth muscle antibodies; and type II, which carries liver, kidney, microsomal antibody. It is probably best to leave classification at this level. Though a theoretical type III has been described associated with soluble liver antigen and possibly induced by drugs, the evidence is not strong. Drug treatment of CAIH remains based on prednisolone, with azathioprine used for steroid-sparing maintenance. If ursodeoxycholic acid has a role it is probably as adjuvant treatment to these.

\section{Viral hepatitis}

The overall results of treatment with interferon alone are not impressive in chronic hepatitis B and $\mathrm{C}$, and the majority of patients are still infected after 6-12 months monotherapy with injections. For hepatitis B patients treatment with lamivudine $100 \mathrm{mg}$ daily has shown promise, and could become a first line option.

In hepatitis $C$ refractory to interferon therapy patients are now offered interferon 3-6 million units three times a week together with ribavirin 1 to $1.2 \mathrm{~g}$ daily. This last drug is recommended only in second line treatment at present, but a case could be made for using it in combination therapy from the outset. It is toxic and regularly produces a $10 \%$ fall in haemoglobin concentration so that close monitoring is necessary and dose reduction may be required. The combination is under review by NICE.

It is expected that the introduction of pegylated interferon will improve treatment in the future.

Whether the long term outcome of hepatitis $B$ virus or hepatitis $C$ virus is affected by treatment is at present unclear. Primary objectives in treatment are disappearance of hepatitis C virus RNA and hepatitis $B$ virus DNA, and conversion of $\mathrm{HBe} \mathrm{Ag}+$ to $\mathrm{HBe} \mathrm{Ag}-$ and $\mathrm{HBe}$ $\mathrm{Ab}+$ status.

\section{Acute intermittent porphyria}

Prevention of known triggers of acute episodes is crucial. When they do occur haem arginate therapy is generally recommended to abort the attack.

\section{Haemochromatosis}

Exact diagnosis depends on liver biopsy, but now it is possible to seek the HFE gene which identifies most but not all patients. Together with serum ferritin, iron, and iron binding capacity and plasma glucose this offers a useful preliminary test in screening relatives of index cases.

\section{Pancreatic insufficiency}

Diagnosis of pancreatic insufficiency is currently based on demonstration of steatorrhoea by triolein breath testing or faecal fat estimation, and collecting evidence of pancreatic enzyme deficiency.

The standard bentiromide test based on pancreatic chymotrypsin therapy is no longer generally available, supplies of bentiromide having become very difficult in the UK. The alternative fluoroscein dilaurate (Pancreolauryl) test is now the standard. This is based on activity of pancreatic esterase. A new test based on the same enzyme is the cholesteryl $-{ }^{14} \mathrm{C}$ octanoyl breath test which shows promise in simplifying investigation.

Serum trypsinogen has also recently been shown to be uniformly low in pancreatic insufficiency.

Where these more sophisticated tests are not available, clinical challenge treatment with pancreatic enzyme supplements in suspected pancreatic insufficiency should lead to reduction in steatorrhoea and weight gain in affected patients. If this test is used it is important formally to demonstrate improvement as steatorrhoea, for example by performing a triolein breath test before and after treatment.

\section{The future}

Over the years various grand ideas have been introduced that were expected to revolutionise the practice of medicine and gastroenterology in particular. Prostaglandins, free oxygen radicals, nitrogen oxide transmitters, and tissue hormones have fascinated scientists and clinicians. The current focus is on molecular biology. Gene probes are available for cystic fibrosis, haemochromatosis, and hereditary colon carcinoma. Regrettably, the long awaited dawn of the age of actual gene therapy in clinical medicine still seems some way off being practical therapeutics.

However, before 1983 nobody thought of peptic ulcer as a curable infectious disease and one looks forward with interest and intrigue to what the future holds.

This survey was based on three main sources:

(1) Systematic review of the journals Gastroenterology, Gut, Hepatology, Alimentary Pharmacology \& Therapeutics, Clinical Gastroenterology, Evidence-Based Medicine, BMF, and the Lancet.

(2) World Congresses of Gastroenterology 1994 and 1998, US Digestive Diseases Week 1995, XIV International Bile Acid Meeting 1996, United European Gastroenterology Weeks 1997 and 1999; and biannual meetings of the British Society of Gastroenterology and the Caledonian Society of Gastroenterology.

(3) Monthly Medline literature reference searches for $\mathrm{H}$ pylori, gall stones, reflux oesophagitis and primary biliary cirrhosis; and participation in the National Barrett's Oesophagus Registry.

\section{Further reading}

1 Medicine 1998;26: issues 7-10 (gastroenterology); 1998;26: issue 11 (nutrition); 1998;26: issue 12 (liver); 1999;27: issue 1 (liver); 1999;27: issue 2 (alcohol).

2 Oxford textbook of hepatology. Oxford: Oxford University Press, 1999.

3 Sleisenger and Fordtran's gastrointestinal and liver disease. Philadelphia: WB Saunders, 1998. 